\section{TATRA \\ MOUNTaiNS \\ Mathematical Publications}

DOI: $10.2478 /$ tmmp-2013-0024

Tatra Mt. Math. Publ. 56 (2013), 35-45

\title{
SOME REMARKS ON PERMUTATIONS WHICH PRESERVE THE WEIGHTED DENSITY
}

\author{
Milan PašTÉKA — ZuZAna VÁClavíKOVÁ
}

\begin{abstract}
In this paper we study the conditions (1), (2) and (3) for the permutations which preserve the weighted density. These conditions are motivated by the conditions of Lévy group, originated in [Levy, P.: Problèmes concrets d'Analyse Fonctionelle. Gauthier Villars, Paris, 1951], and studied in [Obata, N.: Density of natural numbers and Lévy group, J. Number Theory 30 (1988), 288-297]. In the second part we prove that under some conditions for the sequence of weights, for instance for the logarithmic density, the first two conditions can be launched.
\end{abstract}

Let us denote the set of natural numbers by $\mathbb{N}$, and the set of all permutations of the set $\mathbb{N}$ by $\operatorname{Aut}(\mathbb{N})$. Suppose that $\left\{c_{j}\right\}$ is the sequence of positive real numbers such that $\sum_{j=1}^{\infty} c_{j}=\infty$. This sequence will be called the sequence of weights. Let $A \subset \mathbb{N}$. Put

$$
S(A, N)=\sum_{j \leq N, j \in A} c_{j}, S(N)=\sum_{j \leq N} c_{j} .
$$

The value $\bar{d}_{c}(A)=\lim \sup _{N \rightarrow \infty} \frac{S(A, N)}{S(N)}$ we shall call the upper weight density of $A$ and $\underline{d}_{c}(A)=\lim \sup _{N \rightarrow \infty} \frac{S(A, N)}{S(N)}$ we shall call the lower weight density of the set $A$. If there exists $\lim _{N \rightarrow \infty} \frac{S(A, N)}{S(N)}$, then we say that the set $A$ has weighted asymptotic density and we will denote it as $d_{c}(A)$.

The weighted density is object of observations in several works. By $D_{c}$ we will denote the set of all sets, which has the weighted asymptotic density. In special case, if $c_{j}=1$ for all $j$, then the weighted asymptotic density is just asymptotic density and is denoted by $d(A)$.

(C) 2013 Mathematical Institute, Slovak Academy of Sciences. 2010 Mathematics Subject Classification: 11 B05.

Keywords: weighted density, permutation, Levy group.

The research of the first author was supported by the grant VEGA 2/0206/10. 


\section{MILAN PAŠTÉKA — ZUZANA VÁCLAVÍKOVÁ}

$\mathrm{O} b$ at a in $\mathrm{OB}$ ] characterizes the set of permutations of $\mathbb{N}$, which preserve the asymptotic density

$$
G=\left\{g \in \operatorname{Aut}(\mathbb{N}): \lim _{N \rightarrow \infty} \frac{1}{N}|\{1 \leq j \leq N ; g(j)>N\}|=0\right\},
$$

where $\operatorname{Aut}(\mathbb{N})$ is the group of all permutations of $\mathbb{N}$. This set forms a subgroup of the group of all automorphisms of $\mathbb{N}$ with respect to composition and is called the Lévy group.

In the case of weighted asymptotic density, the condition

$$
\lim _{N \rightarrow \infty} \frac{1}{N}|\{1 \leq j \leq N ; g(j)>N\}|=0
$$

splits into two conditions:

$$
\begin{aligned}
& \lim _{N \rightarrow \infty} \frac{1}{S(N)} \sum_{\substack{j \leq N \\
g(j)>N}} c_{j}=0, \\
& \lim _{N \rightarrow \infty} \frac{1}{S(N)} \sum_{\substack{j>N \\
g(j) \leq N}} c_{g(j)}=0,
\end{aligned}
$$

but, as the next example shows, it is not sufficient for preserving the weighted asymptotic density.

EXAMPLE 1. Let the weights be such that $c_{2 k}=1, c_{2 k+1}=0.5$ and let $g \in \operatorname{Aut}(\mathbb{N})$ be such that $g(2 k-1)=2 k$ and $g(2 k)=2 k-1$. Then

$$
\sum_{\substack{j \leq N \\ g(j)>N}} c_{j} \leq 0.5
$$

because just for one element $j \leq N$ the $g(j)$ can exceed the number $N$, so

$$
\lim _{N \rightarrow \infty} \frac{1}{S(N)} \sum_{\substack{j \leq N \\ g(j)>N}} c_{j}=0
$$

Analogously,

$$
\lim _{N \rightarrow \infty} \frac{1}{S(N)} \sum_{\substack{j>N \\ g(j) \leq N}} c_{g(j)}=0 .
$$

But this permutation does not preserve the weighted asymptotic density, because for the set of even numbers $A_{2 k}$ we have $d_{c}\left(A_{2 k}\right)=\frac{2}{3}$ and the image is the set of odd numbers $g\left(A_{2 k}\right)=A_{2 k+1}$ and $d_{c}\left(g\left(A_{2 k}\right)\right)=d_{c}\left(A_{2 k+1}\right)=\frac{1}{3} \neq d_{c}\left(A_{2 k}\right)$. 
We recall the statement well known as

Stolz Theorem. Let $\alpha_{n}, \beta_{n}$ be two sequences of positive real numbers, and $\sum \beta_{n}=\infty$. Then

$$
\lim _{n \rightarrow \infty} \frac{\alpha_{n}}{\beta_{n}}=L \Rightarrow \lim _{N \rightarrow \infty} \frac{\sum_{n \leq N} \alpha_{n}}{\sum_{n \leq N} \beta_{n}}=L .
$$

Let $G_{c}$ be the set of all permutations $g \in A u t(\mathbb{N})$ which satisfy the conditions (11), (2) and

$$
\lim _{j \rightarrow \infty} \frac{c_{g(j)}}{c_{j}}=1
$$

LEMma 1. $G_{c}$ is a group with respect to the composition of permutations.

Pr o of. Clearly, the identic permutation belongs to $G_{c}$, and the set of permutations fulfilling the condition (3) satisfies the conditions of the group. Let $f, g \in G_{c}$. Thus

$$
\begin{gathered}
\lim _{N \rightarrow \infty} \frac{1}{S(N)} \sum_{\substack{j \leq N \\
f(j)>N}} c_{j}=0=\lim _{N \rightarrow \infty} \frac{1}{S(N)} \sum_{\substack{j>N \\
f(j) \leq N}} c_{f(j)}, \\
\lim _{N \rightarrow \infty} \frac{1}{S(N)} \sum_{\substack{j \leq N \\
g(j)>N}} c_{j}=0=\lim _{N \rightarrow \infty} \frac{1}{S(N)} \sum_{\substack{j>N \\
g(j) \leq N}} c_{g(j)} .
\end{gathered}
$$

We prove that $f \circ g$ satisfies (11) and (2). There holds

$$
\begin{aligned}
& \lim _{N \rightarrow \infty} \frac{1}{S(N)} \sum_{\substack{j \leq N \\
(f \circ g)(j)>N}} c_{j}=\lim _{N \rightarrow \infty} \frac{1}{S(N)} \sum_{\substack{j \leq N \\
g(f(j))>N}} c_{j} \\
& =\lim _{N \rightarrow \infty} \frac{1}{S(N)}\left(\sum_{\substack{j \leq N \\
g(f(j))>N \\
f(j) \leq N}} c_{j}+\sum_{\substack{j \leq N \\
g(j(j))>N \\
f(j)>N}} c_{j}\right) \\
& \leq \lim _{N \rightarrow \infty} \frac{1}{S(N)}\left(\sum_{\substack{f(j) \leq N \\
g(f(j))>N}} c_{j}+\sum_{\substack{j \leq N \\
f(j)>N}} c_{j}\right)=\lim _{N \rightarrow \infty} \frac{1}{S(N)} \sum_{\substack{f(j) \leq N \\
g(f(j))>N}} c_{j},
\end{aligned}
$$

for $f, g \in G_{c}$. 


\section{MILAN PAŠTÉKA — ZUZANA VÁCLAVÍKOVÁ}

If the sum

$$
\sum_{\substack{N=1, f(j) \leq N \\ g(f(j))>N}}^{\infty} c_{j}<\infty
$$

then

$$
\lim _{N \rightarrow \infty} \frac{1}{S(N)} \sum_{\substack{f(j) \leq N \\ g(f(j))>N}} c_{j}=0
$$

If the sum $\sum_{N=1, f(j) \leq N, g(f(j))>N}^{\infty} c_{j}=\infty$, then because $\lim _{j \rightarrow \infty} \frac{c_{f(j)}}{c_{j}}=1$, using the Stoltz theorem we have

$$
\lim _{N \rightarrow \infty} \frac{\sum_{\substack{f(j) \leq N \\ g(f(j))>N}} c_{f(j)}}{\sum_{\substack{f(j) \leq N \\ g(f(j))>N}} c_{j}}=1,
$$

and so

$$
\lim _{N \rightarrow \infty} \frac{1}{S(N)} \sum_{\substack{f(j) \leq N \\ g(f(j))>N}} c_{j}=\lim _{N \rightarrow \infty} \frac{1}{S(N)} \sum_{\substack{f(j) \leq N \\ g(f(j))>N}} c_{f(j)}=0,
$$

for $f, g \in G_{c}$. Analogously the second limit is equal to zero.

Now we show that for every $f \in G_{c}$, we have $f^{-1} \in G_{c}$. Let $f \in G_{c}$, so

$$
\lim _{N \rightarrow \infty} \frac{1}{S(N)} \sum_{\substack{j \leq N \\ f(j)>N}} c_{j}=0
$$

and

$$
\lim _{N \rightarrow \infty} \frac{1}{S(N)} \sum_{\substack{j>N \\ f(j) \leq N}} c_{f(j)}=0
$$

Using the $f\left(f^{-1}(j)\right)=j$ we have

$$
\begin{aligned}
& \lim _{N \rightarrow \infty} \frac{1}{S(N)} \sum_{\substack{j \leq N \\
f^{-1}(j)>N}} c_{j} \\
& =\lim _{N \rightarrow \infty} \frac{1}{S(N)} \sum_{\substack{f\left(f^{-1}(j)\right) \leq N \\
f^{-1}(j)>N}} c_{f\left(f^{-1}(j)\right)}=0,
\end{aligned}
$$

for $f \in G_{c}$. Analogously the second limit. 
TheOREM 1. If $g \in G_{c}$, then for any set $S \subset \mathbb{N}$ such that $S \in D_{c}$, i.e., it has the weighted asymptotic density, the set $g(S) \in D_{c}$ and $d_{c}(g(S))=d_{c}(S)$.

Proof. Let us denote $F_{N}^{+}(g)=\{g(j) ; j \leq N \wedge g(j)>N\}$. For any set $S \subset \mathbb{N}$ and any $g \in \operatorname{Aut}(\mathbb{N})$ we have

$$
\{g(j) ; j \leq N \wedge g(j) \in S\} \subseteq F_{N}^{+}(g) \cup(S \cap\{1,2, \ldots, N\}) .
$$

Now let $g \in G_{c}$ and $S \subset \mathbb{N}, S \in D_{c}$. Then the upper weighted asymptotic density

$$
\begin{aligned}
& \bar{d}_{c}\left(g^{-1}(S)\right)=\limsup _{N \rightarrow \infty} \frac{1}{S(N)} \sum_{j \leq N} c_{j} \\
& =\limsup _{N \rightarrow \infty} \frac{1}{S(N)} \sum_{\substack{j \leq N \\
g(j) \in S}} c_{j} \\
& \leq \limsup _{N \rightarrow \infty} \frac{1}{S(N)}\left(\sum_{\substack{j \leq N \\
g(j)>N}} c_{j}+\sum_{\substack{i \leq N, i \in S \\
i=g(j)}} c_{j}\right) \\
& =\limsup _{N \rightarrow \infty} \frac{1}{S(N)}\left(\sum_{\substack{j \leq N \\
g(j)>N}} c_{j}+\sum_{\substack{i \leq N, i \in S \\
i=g(j)}} c_{g^{-1}(i)}\right) \\
& =\limsup _{N \rightarrow \infty} \frac{1}{S(N)} \sum_{\substack{i \leq N \\
i \in S}} c_{g^{-1}(i)},
\end{aligned}
$$

for $g \in G_{c}$. If the sum

$$
\sum_{\substack{N=1 \\ i \leq N, i \in S}}^{\infty} c_{g^{-1}(i)}=\infty
$$

then because $\lim _{i \rightarrow \infty} \frac{c_{i}}{c_{g}-1(i)}=1$, using the Stoltz theorem we have

$$
\lim _{N \rightarrow \infty} \frac{\sum_{i \leq N, i \in S} c_{i}}{\sum_{i \leq N, i \in S} c_{g^{-1}(i)}}=1,
$$

and so it is equal to

$$
\limsup _{N \rightarrow \infty} \frac{1}{S(N)} \sum_{i \leq N, i \in S} c_{i}=\bar{d}_{c}(S) .
$$

Thus

$$
\bar{d}_{c}\left(g^{-1}(S)\right) \leq \bar{d}_{c}(S) .
$$




\section{MILAN PAŠTÉKA — ZUZANA VÁCLAVÍKOVÁ}

Using the fact that $G_{c}$ is a group, we have $g, g^{-1} \in G_{c}$, so we obtain the reverse inequality

$$
\bar{d}_{c}(S)=\bar{d}_{c}\left(g\left(g^{-1}(S)\right)\right) \leq \bar{d}_{c}\left(g^{-1}(S)\right)
$$

and so

$$
\bar{d}_{c}\left(g^{-1}(S)\right)=\bar{d}_{c}(S)
$$

Similarly, for the lower weighted asymptotic density we have $\underline{d}_{c}\left(g^{-1}(S)\right)=\underline{d}_{c}(S)$. Thus if the set $S \in D_{c}$, then the set $g(S) \in D_{c}$, and

$$
\bar{d}_{c}\left(g^{-1}(S)\right)=\bar{d}_{c}(S)=\underline{d}_{c}(S)=\underline{d}_{c}\left(g^{-1}(S)\right), \quad \text { i.e., } d_{c}(S)=d_{c}(g(S)) .
$$

If the sum $\sum_{N=1, i<N, i \in S}^{\infty} c_{g^{-1}(i)}<\infty$, then $d_{c}\left(g^{-1}(S)\right)=0$. In this case the sum

$$
\sum_{\substack{N=1, i \leq N \\ i \in \mathbb{N}-S}}^{\infty} c_{g^{-1}(i)}=\infty,
$$

Using the same way for the set $\mathbb{N}-S$, we obtain

$$
d_{c}(\mathbb{N}-S)=d_{c}\left(g^{-1}(\mathbb{N}-S)\right)=1-d_{c}\left(g^{-1}(S)\right)=1-0=1
$$

and because $d_{c}(\mathbb{N}-S)=1-d_{c}(S)$ we have

$$
d_{c}(S)=0=d_{c}\left(g^{-1}(S)\right) .
$$

Remark 1. We can construct the weights such that every permutation will preserve the weighted asymptotic density for any set $S \in D_{c}$.

LEMMA 2. Let the weights $\left\{c_{n}\right\}$ be such that

$$
\lim _{N \rightarrow \infty} \frac{c_{N}}{\sum_{j=1}^{N} c_{j}}=1 .
$$

Then for any $S \subset \mathbb{N}$ it holds that $S \in D_{c}$ if and only if $S$ is finite or $\mathbb{N}-S$ is finite, and for any permutation $g \in \operatorname{Aut}(\mathbb{N})$, if $S \in D_{c}$, then

$$
g(S) \in D_{c}, \quad \text { and } \quad d_{c}(S)=d_{c}(g(S)) .
$$

Pr o of. Let us suppose that $S$ is infinite and also $\mathbb{N}-S$ is infinite. The sequence

$$
\left\{\frac{\sum_{\substack{j \leq N \\ j \in S}} c_{j}}{c_{N}}\right\}_{N=1}^{\infty}
$$


will tend for $N \in S$ to 1 and for $N \in \mathbb{N}-S$ to 0 . Because the sets $S, \mathbb{N}-S$ are infinite, the 0 and 1 are the cluster points, and so

$$
\underline{d}_{c}(S)=\liminf _{N \rightarrow \infty} \frac{1}{S(N)} \sum_{\substack{j \leq N \\ j \in S}} c_{j}=\liminf _{N \rightarrow \infty} \frac{\sum_{\substack{j \leq N \\ j \in S}} c_{j}}{c_{N}}=0,
$$

and

$$
\bar{d}_{c}(S)=\limsup _{N \rightarrow \infty} \frac{1}{S(N)} \sum_{\substack{j \leq N \\ j \in S}} c_{j}=\limsup _{N \rightarrow \infty} \frac{\sum_{\substack{j \leq N \\ j \in S}} c_{j}}{c_{N}}=1,
$$

and the set $S$ is not measurable. In the case $S$ is finite, then

$$
\underline{d}_{c}(S)=\liminf _{N \rightarrow \infty} \frac{1}{S(N)} \sum_{\substack{j \leq N \\ j \in S}} c_{j}=0=\limsup _{N \rightarrow \infty} \frac{1}{S(N)} \sum_{\substack{j \leq N \\ j \in S}} c_{j}=\bar{d}_{c}(S),
$$

and $d_{c}(\mathbb{N}-S)=1-d_{c}(S)=1$. Analogously for $\mathbb{N}-S$ it is finite. If $g \in \operatorname{Aut}(\mathbb{N})$, then the image of finite set $S$ is finite set $g(S)$ and so $d_{c}(S)=d_{c}(g(S))=0$, analogously for the case $\mathbb{N}-S$ it is finite.

LEMма 3. Let the weights be not increasing and satisfy

$$
\lim _{N \rightarrow \infty} \frac{N c_{N}}{S(N)}=0
$$

Then if for permutation $g \in \operatorname{Aut}(\mathbb{N})$ is $\lim _{j \rightarrow \infty} \frac{c_{g(j)}}{c_{j}}=1$, then

$$
\lim _{N \rightarrow \infty} \frac{1}{\sum_{j \leq N} c_{j}} \sum_{\substack{j \leq N \\ g(j)>N}} c_{j}=0=\lim _{N \rightarrow \infty} \frac{1}{\sum_{j \leq N} c_{j}} \sum_{\substack{j>N \\ g(j) \leq N}} c_{g(j)} .
$$

P r o o f. Let the weights be not increasing and satisfy

$$
\lim _{N \rightarrow \infty} \frac{N c_{N}}{S(N)}=0
$$

Then if there exist the constants $k_{1}, k_{2}$ such that $0<k_{1} \leq \frac{c_{n}}{c_{g(n)}} \leq k_{2}<\infty$, then we have $c_{n} \leq k_{2} c_{g(n)}$, and so

$$
\sum_{\substack{j \leq N \\ g(j)>N}} c_{j} \leq k_{2} \sum_{\substack{j \leq N \\ g(j)>N}} c_{g(j)} \leq k_{2} N c_{N}
$$

because the weights are not increasing. So the

$$
\lim _{N \rightarrow \infty} \frac{1}{S(N)} \sum_{\substack{j \leq N \\ g(j)>N}} c_{j} \leq \lim _{N \rightarrow \infty} \frac{1}{S(N)} k_{2} N c_{N}=0
$$




\section{MILAN PAŠTÉKA — ZUZANA VÁCLAVÍKOVÁ}

Analogously for the second limit we have

$$
0<\frac{1}{k_{2}} \leq \frac{c_{g(j)}}{c_{j}} \leq \frac{1}{k_{1}}<\infty,
$$

so

$$
\begin{gathered}
c_{g(j)} \leq \frac{1}{k_{1}} c_{j}, \\
\sum_{\substack{j>N \\
g(j) \leq N}} c_{g(j)} \leq \frac{1}{k_{1}} \sum_{\substack{j>N \\
g(j) \leq N}} c_{j} \leq \frac{1}{k_{1}} N c_{N},
\end{gathered}
$$

and

$$
\lim _{N \rightarrow \infty} \frac{1}{S(N)} \sum_{\substack{j>N \\ g(j) \leq N}} c_{g(j)} \leq \lim _{N \rightarrow \infty} \frac{1}{S(N)} \frac{1}{k_{1}} N c_{N}=0 .
$$

If the $\lim _{j \rightarrow \infty} \frac{c_{g(j)}}{c_{j}}=1$, then the existence of the constants $k_{1}, k_{2}$ is guaranteed, so the proof is completed.

A special case of weighted asymptotic density is the logarithmic density, where the weights are $c_{j}=\frac{1}{j}$, and the $\lim _{N \rightarrow \infty} \frac{N c_{N}}{S(N)}=\lim _{N \rightarrow \infty} \frac{N \frac{1}{N}}{S(N)}=0$, so the condition of previous lemma is satisfied. The next corollary follows immediately from the lemma.

Corollary 1. Let $d_{c}(A)$ be the logarithmic density and let $g \in A u t(\mathbb{N})$ be a permutation. If $\lim _{j \rightarrow \infty} \frac{c_{g(j)}}{c_{j}}=1$, then $g$ preserves the logarithmic density.

Now we prove a special condition for a specific type of weights. Suppose that the sequence of weights $\left\{c_{n}\right\}$ has moreover two following properties:

For two sequences of positive integers $\{n\},\{k(n)\}$ such that $k(n) \rightarrow \infty, n \rightarrow \infty$ we have

$$
\lim _{n \rightarrow \infty} \frac{n}{k(n)}=1 \Longrightarrow \lim _{n \rightarrow \infty} \frac{c_{n}}{c_{k(n)}}=1
$$

There exists a positive real valued function $\omega(\delta)$ such that $\lim _{\delta \rightarrow 1} \omega(\delta)=1$, and

$$
\lim _{N \rightarrow \infty} \frac{S(\delta N)}{S(N)}=\omega(\delta) .
$$

TheOREM 2. Let the weights $\left\{c_{n}\right\}$ satisfy conditions (41) and (5). Then for every permutation $g: \mathbb{N} \rightarrow \mathbb{N}$ such that

$$
\lim _{n \rightarrow \infty} \frac{g(n)}{n}=1,
$$

and for every set $A \subset \mathbb{N}$ there holds: If $A$ has the weighted density, then also its image $g(A)$ has the weighted density and $d_{c}(A)=d_{c}(g(A))$. 
Pr o of. Let the set $A \subset \mathbb{N}$ have the weighted density. If $A$ is a finite set, then $g(A)$ is also finite and the assertion holds. Suppose that $A$ is an infinite set. Let us choose an arbitrary $\varepsilon>0$. Then the condition (6) implies that

$$
(1-\varepsilon) n \leq g(n) \leq(1+\varepsilon) n, \quad n \geq n_{0}
$$

for suitable $n_{0} \in \mathbb{N}$. The set $A$ can be decomposed into two subsets

$$
A=A_{0} \cup A_{1}, \quad \text { where } \quad A_{0}=A \cap\left[0, n_{0}\right) .
$$

Clearly it holds $d_{c}(A)=d_{c}\left(A_{1}\right), d_{c}\left(g\left(A_{0}\right)\right)=0$. Analogously we have

$$
\bar{d}_{c}\left(g\left(A_{1}\right)\right)=\bar{d}_{c}(g(A)) \quad \text { and } \quad \underline{d}_{c}\left(g\left(A_{1}\right)\right)=\underline{d}_{c}(g(A)) .
$$

There it holds

$$
S\left(g\left(A_{1}\right), N\right)=\sum_{\substack{g(a) \leq N \\ a \in A_{1}}} c_{g(a)} .
$$

From the inequalities (7) we obtain

$$
\sum_{\substack{a \leq \frac{N}{1+\varepsilon} \\ a \in A_{1}}} c_{g(a)} \leq S\left(g\left(A_{1}\right), N\right) \leq \sum_{\substack{a \leq N \\ 1-\varepsilon \\ a \in A_{1}}} c_{g(a)} .
$$

The conditions (44) and (6) imply that $\frac{c_{g(a)}}{c_{a}} \rightarrow 1$ as $a \rightarrow \infty$. Thus if $\sum_{a \in A} c_{a}<\infty$, then also $\sum_{g(a) \in g(A)} c_{g(a)}<\infty$ and $d_{c}(A)=d_{c}(g(A))=0$. Suppose now $\sum_{a \in A} c_{a}=\infty$. From Stolz Theorem we obtain for arbitrary $\delta>0$

$$
\sum_{\substack{a \leq \delta N \\ a \in A_{1}}} c_{g(a)} \sim S\left(A_{1}, \delta N\right), \quad N \rightarrow \infty .
$$

Now from (9) and (5) we have for $\delta>0$

$$
\frac{1}{S(N)} \sum_{\substack{a \leq \delta N \\ a \in A_{1}}} c_{g(a)} \rightarrow \omega(\delta) d(A), \quad N \rightarrow \infty .
$$

And so from (8) we obtain

$$
\omega\left(\frac{1}{1+\varepsilon}\right) d_{c}(A) \leq \underline{d}_{c}(g(A)) \leq \bar{d}_{c}(g(A)) \leq \omega\left(\frac{1}{1-\varepsilon}\right) d_{c}(A) .
$$

Thus for $\varepsilon \rightarrow 0^{+}$the condition (5) yields $d_{c}(A)=d_{c}(g(A))$. 


\section{MILAN PAŠTÉKA — ZUZANA VÁCLAVÍKOVÁ}

$\mathrm{Nath}$ anson and Parikh proved in NP following result:

Theorem A. Let $f: \mathbb{N} \rightarrow \mathbb{N}$ be a one-to-one function such that if the set $A$ of positive integers has asymptotic density, then the set $f(A)$ also has asymptotic density. Let

$$
\lambda=d(f(\mathbb{N})) .
$$

Then for every $A \in \mathcal{D}$ we have

$$
d(f(A))=\lambda d(A)
$$

Using the ideas from theirs proof the following "formal" improvement can be made:

TheOREM B. Let $\mathcal{A}$ be a q-algebra of sets of positive integers and $\nu$ a finitely additive probability measure on $\mathcal{A}$ which has the Darboux property on $\mathcal{A}$. Let $g: \mathbb{N} \rightarrow \mathbb{N}$ be such injective mapping that $g(A) \in \mathcal{A}$ for $A \in \mathcal{A}$ and for $A, B \in \mathcal{A}$ it holds

$$
\nu(A)=\nu(B) \Rightarrow \nu(g(A))=\nu(g(B)) .
$$

Then for every $A \in \mathcal{A}$ we have $\nu(g(A))=\lambda \nu(A)$, where $\lambda=\nu(g(\mathbb{N}))$.

P r o o f. Let $\frac{p}{q} \in\langle 0,1\rangle, p, q \in \mathbb{N}$. Suppose that

$$
A \in \mathcal{A} \quad \text { and } \quad \nu(A)=\frac{p}{q} .
$$

Using the Darboux property we get that there is a decomposition

$$
\mathbb{N}=B_{1} \cup \cdots \cup B_{q}, \quad B_{j} \in \mathcal{A}, \quad \nu\left(B_{j}\right)=\frac{1}{q}, \quad j=1, \ldots, q,
$$

thus

$$
g(\mathbb{N})=g\left(B_{1}\right) \cup \cdots \cup g\left(B_{q}\right), \quad g\left(B_{j}\right) \in \mathcal{A} .
$$

From (i) we obtain $\nu\left(g\left(B_{j}\right)\right)=\frac{\lambda}{q}, j=1, \ldots, q$. The set $A$ can be decomposed into

$$
A=A_{1} \cup \cdots \cup A_{p}, \quad A_{i} \in \mathcal{A}, \quad \nu\left(A_{i}\right)=\frac{1}{q}, \quad i=1, \ldots, p .
$$

Thus we have a decomposition $g(A)=g\left(A_{1}\right) \cup \cdots \cup g\left(A_{p}\right), g\left(A_{i}\right) \in \mathcal{A}$. From (i) we get $\nu\left(g\left(A_{i}\right)\right)=\frac{\lambda}{q}, i=1, \ldots, p$, and so $\nu(g(A))=\lambda \frac{p}{q}$.

Now we shall consider a function $\bar{g}:\langle 0,1\rangle \rightarrow\langle 0, \lambda\rangle$ defined as follows: if $x \in\langle 0,1\rangle$, then there exists $A \in \mathcal{A}$ that $\nu(A)=x$. Put $\bar{g}(x)=\nu(g(A))$. The condition (i) provides that this definition is correct, and from the Dardoux property of $\nu$ on $\mathcal{A}$ we get that $\bar{g}$ is non decreasing on $\langle 0,1\rangle$. We proved that $\bar{g}(x)=\lambda x$ for $x$ rational and so $\bar{g}(x)=\lambda x$ for every $x \in\langle 0,1\rangle$. 


\section{REFERENCES}

[PSV] PAŠTÉKA, M.-ŠALÁT, T.-VISNYAI, T.: Remarks on Buck's measure density and a generalization of asymptotic density, Tatra Mt. Math. Publ. 31 (2005), 87-101.

[OB] OBATA, N.: Density of natural numbers and the Lévy group, J. Number Theory 30 (1988), 288-297.

[NP] NATHANSON, M. B.-PARIKH, R.: Density of sets of natural numbers and the Lévy group, J. Number Theory 124 (2007), 151-157.

[LEV] LEVY, P.: Problemès concrets d'Analyse Fonctionelle, Gauthier Villars, Paris, 1951.

Received January 8, 2012

\author{
Milan Paštéka \\ Department of Mathematics \\ Pedagogical Faculty \\ University of Trnava \\ Priemyselná 4 \\ SK-918-43 Trnava \\ SLOVAKIA \\ E-mail: pasteka@mat.savba.sk \\ Zuzana Václaviková \\ Department of Mathematics \\ Faculty of Science \\ University of Ostrava \\ 30. dubna 22 \\ CZ-701-03 Ostrava 1 \\ CZECH REPUBLIC
}

E-mail: zuzana.vaclavikova@osu.cz 